\title{
The effect of modified relaxation breathing technique on pain anxiety in paients with second-degree burns: A clinical trial study
}

\author{
Mohammad Hosseinzadeh-Karimkoshteh ${ }^{1}(\mathbb{D})$ Mohammadreza Firouzkouhi ${ }^{\mathbb{D}}$, Nosratollah Masinaeinezhad $^{2} \mathbb{D}$, and \\ Abdolghani Abdollahimohammad ${ }^{2, \square},(10)$
}

1 Amiralmomenin Ali Hospital of Zabol, Zabol University of Medical Sciences, Zabol, Iran

2 Medical-Surgical Department, School of Nursing and Midwifery, Zabol University of Medical Sciences, Zabol, Iran

Purpose: To determine the effect of modified relaxation breathing technique on pain anxiety in patients with second-degree burns. Methods: The present study is a clinical trial that was performed on 30 patients with second-degree burns admitted to the Imam Hossein Burn Center in Zabol, Iran. Patients in the experimental group performed breathing technique in the form of 4 counts deep inspiration through the nose, pause for four counts, and expiration with pursed lips for four counts. The experiment was done half an hour for four days before dressing. Data were analyzed using Mann-Whitney U statistical test. The significance level was set at $<0.05$. Results: There was no significant different on pain anxiety between study groups prior to the intervention $(p=0.287)$. However, the median scores of pain anxiety in the experimental group in four sessions were significantly reduced compared to the control group after the intervention $(p<0.001)$. Conclusion: The modified relaxation breathing technique has reduced pain anxiety in patients with second-degree burns. Therefore, it is recommended to be used along with other methods to control pain anxiety for the patients.

Keywords: Anxiety, Bandages, Burns, Iran, Pain anxiety

$\square$ Corresponding: abdollahi@zbmu.ac.ir; Received: October 14, 2020; Accepted: November 9, 2020; Published: January 1, 2021; This article is available from: https://www.jnexp.ir

\section{Introduction}

Pain anxiety is the most common form of anxiety in burn patients and occurs when individuals predict pain in painful procedures such as wound dressing changes [1]. Patients with higher levels of pain anxiety have less pain tolerance and report more pain when changing dressings. High level of anxiety reduces treatment adherence, increases the length of hospitalization, and reduces patients' quality of life [2]. Therefore, controlling pain anxiety is essential for burn patients.

In addition to conventional pharmacological methods, non-pharmacological ones can be used to control pain anxiety; using cognitive-behavioral techniques, for instance, effectively reduce pain anxiety [3]. Breathing techniques are one of the methods of distraction that includes several types such as diaphragmatic breathing, yoga breathing, unilateral nostril breathing, hi-ho rhythmic breathing, and four-count deep regular breathing [4].

Despite significant advances in the management of anxiety, there is still a lack of managing or controlling it in burn patients. Therefore, this study was conducted to determine the effect of deep and regular four-count breathing technique on pre-dressing pain anxiety in patients with second-degree burns.

\section{Methods}

\section{Ethical approval}

The ethical committee of Zabol University of Medical Sciences approved the ethical considerations (IR.ZBMU.REC.1397.037). In order to consider ethical 
issues, the objectives of the study were explained to the patients and the consent form was achieved. .

\section{Study design/Setting}

The present study is a clinical trial study that was conducted in Imam Hossein Burn and Repair Center in Zabol, Iran, in 2018.

\section{Participants}

The research sample consisted of patients who met the inclusion criteria, which included having a second-degree burn on the limbs, being fully conscious, having respiratory stability, having at least $5 \%$ of second-degree burns, having a flame and boiling water burn, and having at least one dressing experience before the experiment. Exclusion criteria included having burned in the chest and abdomen, having a history of anxiety, having intentional and inhalation burns, and death during the study.

The sample size was estimated according to a similar study [5] and based on the formula for comparing means [6] with the alpha level of 0.05 and test power of 0.80. Eligible samples were randomly divided into intervention and control groups by coin toss method.

\section{Data measurement/ collection}

Data collected using a demographic questionnaire and the Burn Patient Anxiety Inventory [7]. The demographics were age, sex, percentage of burns, type of burn, and education level. The BSPAS includes nine items in the four-point Likert scale from never (1) to high (4). A score of 9 indicates no pain anxiety and a score of 36 indicates the highest level of pain anxiety. The method of performing the deep regular four-count breathing was explained and demonstrated by the researcher while the patient was at rest and away from stress. An audio file explaining the steps of the experiment was also played out to patients to coordinate their breathing with it. Patients were included in the study after learning and performing breathing exercises. Patients in the experimental group performed breathing technique in the form of 4 counts deep inspiration through the nose, pause for four counts, and expiration with pursed lips for four counts. The experiment was done half an hour for four days before changing dressings. This method is modified 4-7-8 breathing or relaxation breathing [6].

The experimental group performed the respiratory intervention for 30 minutes and at least 20 respiratory cycles before dressing sessions. The experiment continued for four consecutive days. Data in both groups were collected daily by the researcher only once before changing the dressing. No intervention was performed in the control group except the routine care.

\section{Analyses}

Data analysis was done using SPSS 23 (IBM Co., Armonk, NY, USA). The pain anxiety scores distribution were non-normal based on Shapiro-Wilk test; thus, Mann-Whitney U test was used to compare the median scores between the two groups.

\section{Results}

The mean age of patients were 25.47 ( $\mathrm{SD}=9.55$ and $28.06(\mathrm{SD}=11.38)$ in experimental and control group, respectively. The average burn area was about 19\%. Most of the patients were male in both group and the fire was the main cause of burns [Table 1].

Table 2 shows no significant difference between the scores of pain anxiety based on Mann-Whitney $U$ test in the two groups before the intervention ( $p=0.287$ ). However, a significant difference in median score of pain anxiety between the experimental and control group was found after performing the intervention ( $p s$ $<0.001)$. 
Table 1 demographic profile of patients

\begin{tabular}{lll}
\hline Variable & $\begin{array}{l}\text { Experiment } \\
(\mathbf{n}=\mathbf{1 5})\end{array}$ & $\begin{array}{l}\text { Control } \\
(\mathbf{n}=\mathbf{1 5})\end{array}$ \\
\hline Age (year), Mean (SD) & $25.47(9.55)$ & $28.06(11.38)$ \\
Burn percentage, Mean (SD) & $19.37(8.5)$ & $19.33(11.78)$ \\
Min-Max, \% & $5-45$ & $5-45$ \\
Sex & $\mathbf{n}(\%)$ & $\mathbf{n}(\%)$ \\
Male & $10(66.7)$ & $9(60)$ \\
Female & $5(33.3)$ & $6(40)$ \\
Cause of burn & & \\
Fire & $14(93.3)$ & $10(66.7)$ \\
Hot water & $1(6.7)$ & $5(33.3)$ \\
Education level & & \\
Illiterate & $2(13.3)$ & $1(6.7)$ \\
Under-diploma & $7(46.7)$ & $3(20)$ \\
Diploma & $5(33.3)$ & $11(73.3)$ \\
Academic & $1(6.7)$ & 0 \\
\hline
\end{tabular}

SD, Standard deviation; n, Number;

Table 2 Median and interquartile range of pain anxiety between the experimental and control group

\begin{tabular}{llcl}
\hline $\begin{array}{l}\text { Experiment } \\
\text { Session }\end{array}$ & $\begin{array}{l}\text { Median (Interquartile range) } \\
\text { Experiment } \\
(\mathbf{n = 1 5 )}\end{array}$ & $\begin{array}{l}\boldsymbol{P} \\
\text { Control }\end{array}$ \\
\hline $\mathbf{( n = 1 5 )}$ & \\
\hline $\mathbf{1}$ & $28(6)$ & $30(4)$ & 0.287 \\
$\mathbf{2}$ & $22(6)$ & $30(4)$ & $<0.001$ \\
$\mathbf{3}$ & $20(4)$ & $28(4)$ & $<0.001$ \\
$\mathbf{4}$ & $18(4)$ & $26(4)$ & $<0.001$ \\
\hline
\end{tabular}

\section{Discussion}

The aim of this study was to determine the effect of modified relaxation breathing on the level of pain anxiety before dressing in patients with second-degree 2 burns. The pain anxiety was reduced significantly in the experimental group compared to the control one after performing modified breathing technique. There was a decreasing trend in pain anxiety in both groups over four days, but the downward trend was more in the experimental group. The results indicate the effectiveness of the modified relaxation breathing technique on pain anxiety before dressing in patients with second-degree burns.
Breathing and emotions are interrelated. Increased breathing leads to stress and anxiety. In contrast, deep, slow, and regular breathing with an effect on the autonomic nervous system and cellular homeostasis physiologically inhibits negative emotions such as stress and anxiety [2]. Focusing on an internal factor such as breathing and consciously avoiding other mental and environmental stimuli helps to relax. In the breathing techniques, the activity of the parasympathetic system increases and the activity of the sympathetic system decreases, consequently relaxation happens [9]. Besides, reducing the level of anxiety might be due to decreasing pain levels and adaptive strategies [2].

The effectiveness of breathing techniques to control anxiety have been approved in various groups, including, burn patients [10], women with multiple sclerosis [11], obstetrics [12], coronary artery disease [13], asthmatic patients [14], and prostate biopsy [15]. Previous studies have used a variety of techniques such as diaphragmatic breathing, controlled breathing, yogic breathing, relaxation breathing, unilateral nostril breathing, and a changing breathing pattern [16].

\section{Limitations}

The results of this study might be influenced by other factors such as differences in dressing techniques, race, and social support. The dressing techniques are different for each patient's need and nurses' skills and character. People who do not receive adequate social support have higher levels of stress and anxiety. As social or family support are unequal, the patient probably had different pain anxiety that was beyond the researcher's control. Therefore, the generalizability of results are limited.

\section{Conclusion}

Using the modified relaxation breathing technique is an effective method for pain anxiety in patients with 
second-degree burns. Therefore, it can be used to control pain anxiety in burn patients. This simple, cost-effective technique with unknown side effects can be used as the first line care of treatment to control pain anxiety in the burn patients. On the other hand, in addition to applying the results in the clinical settings, the research results can be used in nursing education and research. Nurses and nursing students can introduce and apply this method for themselves and the others for controlling pain anxiety. Researchers can use the results of this study as a basis for future research, such as the effect of modified relaxation breathing on sleep quality in the burn patients.

\section{Author contributions}

Conceptualization: AA, MF, MK. Formal analysis: AA. Methodology: AA, MF, MK. Writing - original draft: MK, NM. Writing - review \& editing: AA, MF.

\section{Conflict of interest}

No declared.

\section{Funding}

None.

\section{Acknowledgments}

None.

\section{References}

[1] Fakhar FM, Rafii F, Orak RJ. The effect of jaw relaxation on pain anxiety during burn dressings: randomized clinical trial. Burns 2013;39:61-7.

[2] Cimpean A, David D. The mechanisms of pain tolerance and pain-related anxiety in acute pain. Health Psychol Open 2019 Nov 29;6(2):2055102919865161. doi: $10.1177 / 2055102919865161$.

[3] Bandelow B, Michaelis S, Wedekind D. Treatment of anxiety disorders. Dialogues Clin Neurosci 2017 Jun;19(2):93-107. doi:10.31887/DCNS.2017.19.2/bbandelow.
[4] Hoseinzadeh-Karimkoshteh M, Firouzkouhi M, Abdollahimohammad A. Effect of regular smooth breathing on the intensity of pain caused by dressing change in patients with second-degree burns: A Clinical Trial. Med Surg Nurs J 2019; 8(2):e92321. doi: 10.5812/msnj.92321.

[5] Ghadimi Gili E, Fathi M, Kraskian A, Ahadi H. Evaluation of the effect of hypnosis on the anxiety reduction in burn dressing change. Med J Mashhad Univ Med Sci 2016;59(3):163-70.

[6] Abdollahimohammad A, Firouzkouhi M. Sample size estimation in randomized clinical trials (RCTs). J Diabetes Nurs 2019; 7 (1) :737-739. http://jdn.zbmu.ac.ir/article-1-353-fa.html

[7] Taal LA, Faber AW, van Loey NE, Reynders CL, Hofland HW. The abbreviated burn specific pain anxiety scale: a multicenter study. Burns 1999 Sep;25(6):493-7. doi: 10.1016/s03054179(99)00034-0.

[8] Pace-Schott EF, Amole MC, Aue T, Balconi M, Bylsma LM, Critchley H, et al. Physiological feelings. Neurosci Biobehav Rev 2019 Aug;103:267-304. doi: 10.1016/j.neubiorev.2019.05.002.

[9] Russo MA, Santarelli DM, O'Rourke D. The physiological effects of slow breathing in the healthy human. Breathe (Sheff) 2017 Dec;13(4):298-309. doi: 10.1183/20734735.009817.

[10] Park E, Oh H, Kim T. The effects of relaxation breathing on procedural pain and anxiety during burn care. Burns 2013 Sep;39(6):1101-6. doi:10.1016/j.burns.2013.01.006.

[11] Aghili SM, Afzali S. the effect of yoga yoga breathing exercises on chronic low pain, anxiety, psychological and physical wellbeing of women with MS. Q J HEALTH PSYCHOL 2017; 5(20): 109-124.

[12] Baljon KJ, Romli MH, Ismail AH, Khuan L, Chew BH. Effectiveness of breathing exercises, foot reflexology and back massage (BRM) on labour pain, anxiety, duration, satisfaction, stress hormones and newborn outcomes among primigravidae during the first stage of labour in Saudi Arabia: a study protocol for a randomized controlled trial. BMJ Open 2020 June 15;10(6):e033844. doi:10.1136/bmjopen-2019-033844.

[13] Amjadian M, Bahrami Ehsan H, Saboni K, Vahedi S, Rostami R, Roshani D. A pilot randomized controlled trial to assess the effect of Islamic spiritual intervention and of breathing technique with heart rate variability feedback on anxiety, depression and psycho-physiologic coherence in patients after coronary artery bypass surgery. Ann Gen Psychiatry 2020 August 13;19:46. doi:10.1186/s12991-020-00296-1.

[14] Dhooria S, Agarwal R. Controlling asthma by breathing techniques: Role of anxiety. Chest 2015 Mar;147(3):e130. doi: 10.1378/chest.14-2755.

[15] Grinberg AS, Sellinger JJ, Sprenkle PC, Bandin AJ, Nawaf CB, Syed JS, Leapman MS. Effect of Diaphragmatic Breathing on Procedural Anxiety During Transrectal Prostate Biopsy. Urology 2020 Mar;137:26-32. doi: 10.1016/j.urology.2019.12.024. Nivethitha L, Mooventhan A, Manjunath NK. Effects of various prānāyāma on cardiovascular and autonomic variables. Anc Sci Life 2016 Oct-Dec;36(2):72-77. doi: 10.4103/asl.ASL_178_16.

“Cite: Hosseinzadeh-Karimkoshteh M, Firouzkouhi M, Masinaeinezhad N, Abdollahimohammad A. The effect of modified relaxation breathing technique on pain anxiety in patients with second-degree burns: A clinical trial study. $J$ Nurs Explor 2021 Jan;1(1):11 . https://doi.org/10.29252/jnexp.1.1.11 